\title{
Gastric Leiomyoma Misdiagnosed as a Left Cystic Adrenal Tumor
}

\author{
Young-Joo Kim \\ Department of Urology, School of Medicine, Jeju National University, Jeju, Korea
}

\begin{abstract}
Gastrointestinal leiomyomas often occur in the esophagus, colon, and rectum, but are very rare in the stomach. The patient was referred to Jeju national university medical center for the treatment of a left adrenal tumor diagnosed 5 years ago. She had a long history of repeated left flank pain, nausea, and abdominal distension for the last few years. Annual follow-up computed tomography scan showed a no changed $5 \times 5.5-\mathrm{cm}$ sized multiloculated left adrenal cystic lesion. Laboratory tests revealed that the basal levels of plasma adrenocorticotropin, cortisol, aldosterone, renin, testosterone, adrenaline, noradrenaline, and dopamine were normal findings. The preoperative diagnosis was a left nonfunctioning adrenal neoplasm with symptom of discomfort. However, the tumor revealed as an exophytic growing leiomyoma with cystic changes of a stomach by surgery. The mass was found at the posterior wall of the fundus and about 5-cm-sized mass. Resection of the tumor was performed using laparoscopy. The pathologic diagnosis revealed a gastrointestinal leiomyoma with a benign cystic lesion lined by respiratory epithelium with an old hemorrhage and chronic gastritis. The patient had no evidence of recurrence during the 12-month follow-up. Gastric leiomyoma is unfamiliar to the usual urologists. It needs to take gastric leiomyoma into account if the left adrenal tumor is close to the stomach. Herein, we report the case of a 44-year-old woman with gastrointestinal leiomyoma misdiagnosing as a nonfunctioning adrenal tumor.
\end{abstract}

Key Words: Leiomyoma, Adrenal gland, Neoplasms, Laparoscopy
Received May 17, 2021

Revised July 11, 2021

Accepted July 19, 2021

Corresponding author:

Young-Joo Kim

Email: kurology@jejunu.ac.kr

https://orcid.org/0000-0001-5580-4338

This work was supported by the 2021 education, research and student guidance grant funded by Jeju National University.
Gastrointestinal leiomyomas are rare benign gastrointestinal tract tumors in the stomach especially fundus, but found common in esophagus, colon, and rectum. However, there have been few reports of exophytic pedunculated leiomyoma with cystic changes at gastric fundus. The differential diagnosis will include left adrenal mass, pancreatic and renal cysts as well as duplication cysts. Computed tomography (CT) images of gastric leiomyoma usually have no specific characteristics, and often can be misdiagnosed as soft tissue mass with enhancement. Therefore, a precise preoperative diagnosis of gastric mass is significant, so that an unnecessary exploratory operation can be avoided. The most appropriate treatment for leiomyoma of stomach is surgical resection. The definitive diagnosis of the disease is mainly confirmed by postoperative pathological results and immunochemistry. Here, we report a 44-year-old woman who was diagnosed adrenal nonfunctioning cystic tumor with left flank pain. The tumor was diagnosed as an exophytic pedunculated cystic leiomyoma of the fundus with chronic gastritis. 


\section{CASE REPORT}

A 44-year-old female patient presented with discomfort in the left upper quadrant (LUQ) and was admitted to hospital. On physical examination, no abnormal findings were found. She had a long history of nonfunctioning adrenal cyst and repeated follow-up CT scan annually. The patient suffered from LUQ pain at each meal. There was no abnormal lesion on gastroscopy by annual check-up. CT scan found a $5 \times 5.5-\mathrm{cm}$ multiloculated and a lower density cystic mass in adrenal area (Fig. 1). During enhancement scanning, the tumor was poor enhanced. Based on these results, an initial clinical diagnosis of adrenal nonfunctioning cystic tumor was made. The patient was planned to undergo surgery with laparoscopic adrenalectomy for the persisting LUQ pain. During the surgery, it was easy to find a cystic mass at the upper pole of the left kidney. Since a tumor is hardly distinguishable from adrenal glands, we have resected the tumor as well as the surrounding glandular tissue. But the tumor appeared to be adhering to the fundus of stomach. So, we had a surgical consultation with a general surgeon. Clinical diagnosis of gastrointestinal stromal tumor (GIST) was made. After discussing the case with the surgery team, the patient underwent a laparoscopic hemigastrectomy. Histopathological examination showed leiomyoma with epithelioid cells with old hemorrhage. Immuno-histochemical examination showed that cytoplasm of the tumor was negative for the c-kit protein (CD117) and positive for the superior mesenteric artery (SMA), desmin, and S-100 (Fig. 1). The tumor was diagnosed as an exophytic pedunculated leiomyoma of the fundus with cystic changes. During the 12-month followup examination, the patient has no discomfort symptoms and no recurrence detected in radiology.

\section{DISCUSSION}

Leiomyoma of stomach is a submucosal tumor that
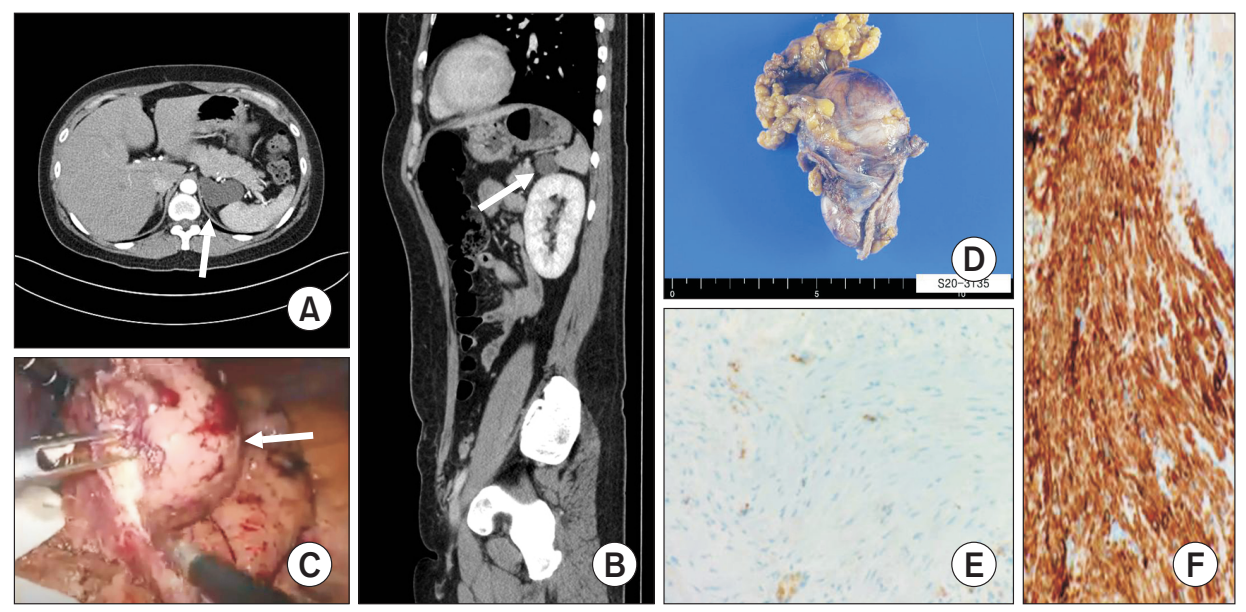

Fig. 1. (A) Axial image of abdominal computed tomography showing a 5.5-cm-sized multiloculated cystic tumor lesion (arrow) in left adrenal gland. (B) Saggital image of abdominal computed tomography showing a $5.5-\mathrm{cm}$-sized multiloculated cystic tumor lesion (arrow) in left adrenal gland. (C) Image of laparoscopic removal of mass (arrow). The isolated cystic mass appeared to be adhering to the fundus of stomach. (D) Gross pathology finding of laparoscopic hemigastrectomy, a cystic leiomyoma ( $5.8 \mathrm{~cm} \times 4.5 \mathrm{~cm} \times 2.5 \mathrm{~cm}$ in dimension) including gastric wall. The outer surface shows focally attached adipose tissue. (E) On immunohistochemical examination, Positive for smooth muscle myosin heavy chain $(\times 400)$. (F) On immunohistochemical examination, the cells of the lesion were negative for CD117 (×400) (c-kit). 
can grow within or outer lumen and represent $2.5 \%$ of gastric tumors. ${ }^{1}$ Generally, most gastric leiomyomas have no specific symptoms. Sometimes, they can reveal clinical symptoms such as a hemorrhage from inflammation of the gastric mucosa. ${ }^{2}$ Leiomyomas are the most common tumor like as if histopathology was normal smooth muscle cells in esophagus. They occur very rarely in the stomach. ${ }^{3}$ There were few studies that described similar cases of misdiagnosed gastric mass. Most studies were case reports of male patients with an initial CT scan to visualize the gastric mass in the left adrenal area. Differential diagnoses include left adrenal mass, pancreatic and renal cysts, and overlapping cysts. If diverticulum consists of gas, it may be mistaken for an abscess or necrotic tumor. ${ }^{4}$ The final pathological analysis showed a gastrointestinal leiomyoma of stomach. Clinical findings rely on the size, location, and characteristics of the tumor. Complication related with gastric leiomyoma is discharged in peritoneum due to mucosal ulceration, stomach volvulus, gastric mass torsion, and bleeding. ${ }^{5}$ Homogenous low attenuation and poor enhancement were characteristic of gastric leiomyomas. ${ }^{6}$ More than $90 \%$ of GIST showed a form of peripheral contrast enhancement due to hemorrhage, necrosis cystic changes within the tumor. Less than $10 \%$ showed homogenous enhancement. ${ }^{7}$ CT images of gastric leiomyoma usually have no specific characteristics, and often can be misdiagnosed as soft tissue mass with enhancement. Therefore, accurate diagnosis of gastric mass before surgery is important, so that an unnecessary exploratory operation can be avoided. In the present case, the nonfunctioning cystic adrenal tumor was diagnosed in the initial diagnosis. However, a rereading of CT images identified a tumor closely related to the fundus of the stomach. Therefore, misdiagnosis of nonfunctioning adrenal tumors is also common, so preoperative diagnosis of the fundus leiomyoma requires careful reading of imaging data and much experience in clinical diagnosis. The definitive diagnosis of the disease is mainly confirmed by postoperative pathological results and immunochemistry. On immunohistochemical studies, leiomyomas are mostly positive for desmin and SMA and negative for CD34 and CD117 (c-kit) in almost every case. ${ }^{8}$ Leiomyoma in our study showed negative finding for CD34 and CD117 (c-kit), and positive finding for SMA and desmin. The most appropriate treatment for leiomyoma of stomach is surgical resection. The most common location of gastric leiomyomas is the cardia of stomach. ${ }^{9}$ The cardial location of the tumors has significant clinical importance. Minimal invasive surgical methods such as endoscopic resection or laparoscopic wedge resection have developed in gastric tumor treatment. ${ }^{10}$ An multidetector CT scanner with the capability for very thin slice thickness and multiplanner reformation images using intravenous contrast media or additional magnetic resonance imaging proved to be very a useful method in the assurance of gastric mass and in differentiating them from other lesions particularly adrenal tumors or pseudo-tumors. It is important to distinguish leiomyoma from other malignancies such as leiomyosarcomas and GISTs. We experienced a case of gastric leiomyoma mimicking left adrenal cystic mass with symptoms. which led us to the wrong treatment during the perioperative period. Gastric leiomyoma is unfamiliar to the usual urologists. It needs to take gastric leiomyoma into account if left adrenal tumor is close to the stomach.

\section{CONFLICT OF INTEREST}

The authors claim no conflicts of interest. 


\section{REFERENCES}

1. Miettinen M, Lasota J. Gastrointestinal stromal tumors: pathology and prognosis at different sites. Semin Diagn Pathol 2006;23:70-83.

2. Daryl R, Qiuxue T, Tan Sofia N, Emmanuel O, Denzil E, Madhavi R. Ulcerated gastric leiomyoma causing massive upper gastrointestinal bleeding: a case report. Mol Clin Oncol 2018;8:671-4.

3. Miettinen M, Sarlomo-Rikala M, Sobin LH, Lasota J. Esophageal stromal tumors: a clinicopathologic, immunohistochemical and molecular genetic study of 17 cases and comparison with esophageal leiomyomas and leiomyosarcomas. Am J Surg Pathol 2000;24:211-22.

4. Schwartz AN, Goiney RC, Graney DO. Gastric diverticulum simulating an adrenal mass: $\mathrm{CT}$ appearance and embryogenesis. AJR Am J Roentgenol 1986;146:553-4.

5. Masayoshi Y, Ryoji K, Ichiro O, Kaveh M, Satoru N, Haruhisa S, et al. Different histological status of gastritis in superficial adenocarcinoma of the esophagogastric junction. Jpn J Clin Oncol 2014; 44:65-71.

6. Lee MJ, Lim JS, Kwon JE, Kim H, Hyung WJ, Park MS, et al. Gastric true leiomyoma: computed tomographic findings and pathological correlation. J Comput Assist Tomogr 2007;31:204-8.

7. Levy AD, Remotti HE, Thompson WM, Sobin LH, Miettinen M. Gastrointestinal stromal tumors: radiologic features with pathologic correlation. Radiographics 2003;23:283-304.

8. Liu Q, Wang Y, Kong L, Kan Y. Study on clinicopathological features of gastrointestinal stromal tumor and relevant prognostic factors. Cell Biochem Biophys 2015;73:743-7.

9. Miettinen M, Lasota J. Gastrointestinal stromal tumors: review on morphology, molecular pathology, prognosis, and differential diagnosis. Arch Pathol Lab Med 2006;130:1466-78.

10. Rutkowski P, Hompes D. Combined therapy of gastrointestinal stromal tumors. Surg Oncol Clin N Am 2016;25:735-59. 